\title{
Correction to: Impact of Enhanced Recovery After Surgery on Postoperative Outcomes for Patients Undergoing Cytoreductive Surgery and Hyperthermic Intraperitoneal Chemotherapy
}

\section{Bradley White, $\mathrm{MD}^{1}$, Fadi Dahdaleh, $\mathrm{MD}^{2}$, Samer A. Naffouje, $\mathrm{MD}^{3}$, Neerav Kothari, $\mathrm{MD}^{4}$,} Jessica Berg, $\mathrm{PA}^{2}$, Wendy Wiemann, $\mathrm{MA}^{2}$, and George I. Salti, $\mathrm{MD}^{1,2}$

${ }^{1}$ Department of General Surgery, Division of Surgical Oncology, The University of Illinois at Chicago, Chicago, IL ; ${ }^{2}$ Department of Surgical Oncology, Edward-Elmhurst Health, Naperville, IL; ${ }^{3}$ Department of Surgical Oncology, H. Lee Moffitt Cancer Center, Tampa, FL; ${ }^{4}$ Department of Anesthesia, University of Illinois Hospital and Health Sciences System, Chicago, IL

\section{CORRECTION TO:}

ANN SURG ONCOL

HTTPS://DOI.ORG/10.1245/S10434-020-09476-5

The following Acknowledgment is missing from the original article.
ACKNOWLEDGMENT The authors acknowledge the contribution to the article of Drs. Tom D. Beris and Ryan Vealey, DuPage Valley Anesthesiologists, Ltd, who contributed to the article in the following way: ERAS protocol.

The original article can be found online at https://doi.org/10.1245/ s10434-020-09476-5.

(C) Society of Surgical Oncology 2021

Published Online: 19 February 2021

G. I. Salti, MD

e-mail: geosalti@uic.edu 